\title{
Simulation training using WhatsApp (Sim-thru-WhatsApp) improves doctors' confidence in endocrine and diabetes case management
}

\author{
Authors: Wentin Chen, ${ }^{A}$ Punith Kempegowda, ${ }^{B}$ Eka Melson, ${ }^{B}$ Meri Davitadze, ${ }^{C}$ Manal Aftab, ${ }^{D}$ Emma Ooi, ${ }^{E}$ \\ Cai Ying Ng, ${ }^{\mathrm{E}}$ Parisha Blaggan, ${ }^{\mathrm{A}}$ Thia Hanania, ${ }^{\mathrm{A}}$ Lucretia Thomas, ${ }^{\mathrm{A}}$ Dengyi Zhou, ${ }^{\mathrm{A}}$ Sailesh Sankar, ${ }^{\mathrm{F}}$ John Ayuk ${ }^{\mathrm{D}}$ \\ and Muhammad Ali Karamat ${ }^{\mathrm{D}}$
}

\section{Introduction}

Simulation is defined as a process that substitutes or amplifies real patient encounters with artificial models, live actors, or virtual reality patients. ${ }^{1}$ The goal of simulation is to replicate patient care scenarios with guided experiences in a realistic environment. ${ }^{2}$ This is usually followed by debriefing sessions to facilitate abstraction, conceptualisation and relation to clinical practice, in order to promote higher learner retention. ${ }^{3}$ Simulation creates an ideal educational environment as learning activities are standardised, safe, and reproducible, and knowledge, skills and attitudes can be acquired in a safe and efficient manner. ${ }^{2,4}$

Simulation-based learning (SBL) has increasingly been used as a learning tool in undergraduate and postgraduate medical curricula, to help the modern healthcare professional to achieve higher levels of competence and safer patient care. ${ }^{4}$ The aim of Sim-thru-WhatsApp is to create a feasible and cost-effective SBL model in medical education. This study was conducted as part of the continuous professional development for Health Education West Midlands specialty trainees in diabetes and endocrinology. It aims to evaluate the effectiveness and acceptance rate of this new SBL model.

\section{Materials and methods}

All specialist registrars who attended the July and October 2019 endocrinology and diabetes training days, organised by Health Education West Midlands, participated in the study. Standardised transcripts of five anonymised pituitary cases (non-functioning pituitary adenoma,

Authors: ${ }^{A}$ University of Birmingham Medical School, Birmingham,

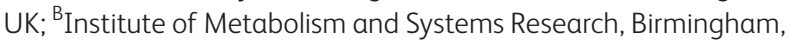
UK; ' Georgian-American Family Medicine Clinic 'Medical House', Tbilisi, Georgia; ' University Hospitals Birmingham NHS Foundation

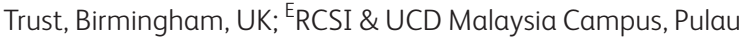
Pinang, Malaysia; ${ }^{{ }^{F}}$ Health Education England (West Midlands), Birmingham, UK craniopharyngioma, macroprolactinoma, acromegaly and Cushing's disease) were used for the July training day. Five diabetes cases (hypoglycaemic unawareness and insulin pump, monogenic diabetes during pregnancy, interpreting Libre, dawn phenomenon, hypoglycaemic unawareness and islet transplant) were used for the October training day. These scenarios were based on real-life cases and approved by relevant experts prior to their inclusion. Moderators, specifically trained on these scenarios, interacted with trainees through WhatsApp. The diabetes simulation was preceded by a lecture covering the basics of diabetes technology. All cases were then discussed by a consultant, with reference to local and national guidelines. Trainee acceptance rate and confidence levels pre- and post-simulation of simulated cases vs non-simulated cases were compared by assessing the Chisquared statistics.

\section{Results and discussion}

Twenty-four and 17 trainees participated in the endocrine and diabetes simulation respectively. There were significant improvements in trainees' confidence levels in managing craniopharyngioma $(p=0.0179)$ and acromegaly $(p=0.0025)$, and interpreting continuous glucose monitor readings $(p=0.02)$. There was a trend towards increased confidence in managing macroprolactinoma, Cushing's disease, hypoglycaemic unawareness, monogenic diabetes during pregnancy and interpreting Libre readings. Overall, trainees' confidence improved significantly in simulated endocrine and diabetes case scenarios ( $p=0.0002$ and $p<0.01$, respectively). In comparison, there were no significant changes in confidence levels for non-simulated cases. Trainees also reported that Sim-thru-WhatsApp accommodated their learning styles, was engaging and worth their time.

\section{Conclusion}

Sim-thru-WhatsApp proved to be an effective teaching model, with a high acceptance rate, which improved doctors' confidence in managing endocrine and diabetes cases. Further sessions with other conditions and specialities are necessary to 
assess the wider application of Sim-thru-WhatsApp in medical training.

\section{Conflicts of interest}

None declared.

\section{References}

1 Gaba DM. The future vision of simulation in health care. Qual Saf Health Care 2004;13:2-10.

2 Okuda Y, Bryson EO, DeMaria S Jr et al. The utility of simulation in medical education: what is the evidence? Mt Sinai J Med 2009;76:330-43.

3 So HY, Chen PP, Wong GKC, Chan TTN. Simulation in medical education. J R Coll Physicians Edinb 2019;49:52-7.

4 Aggarwal R, Mytton OT, Derbrew M et al. Training and simulation for patient safety. Qual Saf Health Care 2010;19:34-43. 